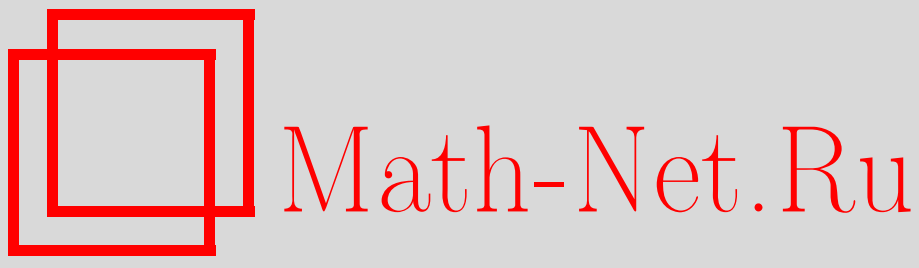

В. Л. Колмогоров, В. П. Федотов, Л. Ф. Спевак, Н. А. Бабайлов, В. Б. Трухин, Решение нестационарных температурных и термомеханических задач методом разделения переменных в вариационной постановке, Вестн. Сам. гос. техн. ун-та. Сер. Физ.-мат. науки, 2006, выпуск 42, 72-75

DOI: https://doi.org/10.14498/vsgtu413

Использование Общероссийского математического портала Math-Net.Ru подразумевает, что вы прочитали и согласны с пользовательским соглашением http://www . mathnet.ru/rus/agreement

Параметры загрузки:

IP : 3.80 .253 .173

26 апреля 2023 г., 16:45:24

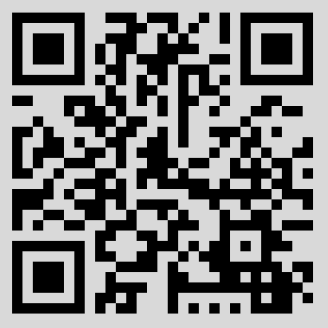




\section{РЕШЕНИЕ НЕСТАЦИОНАРНЫХ ТЕМПЕРАТУРНЫХ И ТЕРМОМЕХАНИЧЕСКИХ ЗАДАЧ МЕТОДОМ РАЗДЕЛЕНИЯ ПЕРЕМЕННЫХ В ВАРИАЦИОННОЙ ПОСТАНОВКЕ}

Предложены алгоритмы решения нестационарных тепловых и связных термомеханических задач методом разделения переменных, основанных на вариационной постановке краевых задач.

В работах $[1,2]$ предложен метод решения нестационарных задач математической физики, основанный на совместном применении вариационных принципов и метода разделения переменных. Суть метода состоит в следующем. Для задачи определения напряженнодеформированного состояния или задачи теплопроводности формулируются вариационные принципы, соответствующие фиксированному моменту времени $t$. Искомые функции (скорости, напряжения, температура) представляются в виде отрезков рядов по известным координатным функциям, коэффициенты при которых - неизвестные функции времени. В каждый момент времени эти коэффициенты считаются варьируемыми параметрами. Координатные функции задаются исходя из граничных условий задачи. Подставляя отрезки рядов в вариационные принципы, и записывая необходимые условия экстремума относительно варьируемых параметров, мы получаем систему обыкновенных дифференциальных уравнений относительно неизвестных функций времени. Решение системы дает решение задачи для любого момента $t$ в рамках исходной постановки. В работах $[3,4]$ предложенным методом успешно решен ряд задач деформирования с учетом разрушения и фрагментации. В данной работе представлены алгоритмы применения метода для решения нестационарных задач теплопроводности, а также связных термомеханических задач.

Алгоритм решения задачи теплопроводности. Рассмотрим нестационарную задачу теплопроводности, состоящую в определении в некоторой области $V$ для произвольного момента времени $t>t_{0}$ температуры $\theta$, удовлетворяющей уравнению:

$$
c \rho \frac{d \theta}{d t}=\lambda \Delta \theta+f,
$$

и краевым условиям

$$
\begin{array}{cc}
\text { на поверхности } S_{1}: & \theta=\theta_{*}, \\
\text { на поверхности } S_{2}: & -\lambda \frac{\partial \theta}{\partial n}=\varphi_{*}, \\
\text { на поверхности } S_{3}: & -\lambda \frac{\partial \theta}{\partial n}=\alpha\left(\theta-\theta_{c}\right), \\
\left.\theta\right|_{t=t_{0}}=\theta_{0} .
\end{array}
$$

Здесь $c$ - удельная теплоемкость; $\lambda$ - коэффициент теплопроводности; $\Delta-$ оператор Лапласа; $\alpha-$ коэффициент теплообмена; $\theta_{c}$ - температура среды; $f, \theta_{*}$ и $\varphi_{*}-$ заданные функции; $\theta_{0}-$ начальное распределение температуры. Вариационный принцип, эквивалентный задаче (1)-(2), в фиксированный момент времени выглядит следующим образом:

$$
\delta J_{\theta}=\delta\left\{\int_{V}\left[\frac{\lambda}{2}\left(\nabla \theta^{\prime}\right)^{2}-f \theta^{\prime}+c \rho \oint \theta^{\prime}\right] d V+\int_{S_{2}} \varphi_{*} \theta^{\prime} d S+\alpha \int_{S_{3}}\left(\frac{1}{2} \theta^{\prime 2}-\theta^{\prime} \theta_{c}\right) d S\right\}=0 .
$$

Штрихом отмечена варьируемая функция температуры, $\&$ - ее неварьируемая производная по времени.

В соответствии с методом разделения переменных искомая функция представляется в виде отрезка ряда:

$$
\theta^{\prime}=\sum_{k=1}^{n} a_{k} \theta_{k}(\mathbf{x})
$$

где $\theta_{k}(\mathbf{x})$ - подходящие координатные функции, $a_{k}$ - функции времени, в фиксированный момент времени являющиеся варьируемыми параметрами. 
Функционал $J_{\theta}$ с учетом представления температуры (5) является в фиксированный момент времени $t$ функцией параметров $a_{k}$. Подставляя в необходимые условия экстремума этой функции $\frac{\partial J_{\theta}}{\partial a_{k}}=0, k=1,2, \ldots, n$, значение $\oint_{k}=\sum_{k=1}^{n} \frac{d a_{k}}{d t} \theta_{k}(\mathbf{x})$, получим систему дифференциальных уравнений относительно функций $a_{k}$, решение которой дает распределение температуры в произвольный момент времени $t>t_{0}$.

Описанным методом была решена задача выравнивания температуры по сечению цилиндрической стальной заготовки, находящейся в условиях тепловой изоляции. Задача (1)-(3) имеет в этом случае вид

$$
c \rho \frac{d \theta}{d t}=\lambda \Delta \theta,-\left.\lambda \frac{\partial \theta}{\partial n}\right|_{r=R}=0, \quad \theta_{\substack{t=t_{0} \\ r=0}}=T_{1}, \quad \underset{\substack{t=t_{0} \\ r=R}}{\theta}=T_{2} .
$$

Функционал $J_{\theta}$ для задачи (6) в полярной системе координат записывается так:

$$
J_{\theta}=\int_{0}^{R}\left(\frac{\lambda}{2}\left(\frac{\partial \theta^{\prime}}{\partial r}\right)^{2}+c \rho \circledast \theta^{\prime}\right) r d r .
$$

Примем виртуальное температурное поле, удовлетворяющее заданному граничному условию, в следующем виде:

$$
\theta^{\prime}=a\left(2 r^{3}-3 R r^{2}\right)+b,
$$

причем в момент $t=t_{0}$ начальным условиям задачи (6) будут соответствовать следующие начальные условия для параметров $a$ и $b$ :

$$
a\left(t_{0}\right)=a_{0}=-\left(T_{2}-T_{1}\right) / R^{3}, b\left(t_{0}\right)=b_{0}=T_{1} .
$$

Подставляя выражение (8) в функционал (7), а затем заменяя в необходимых условиях экстремума $\partial J_{\theta} / \partial a=0, \partial J_{\theta} / \partial b=0$ соответствующее значение производной:

$$
\oint=\frac{d a}{d t}\left(2 r^{3}-3 R r^{2}\right)+\frac{d b}{d t},
$$

получаем систему дифференциальных уравнений относительно функций $a(t)$ и $b(t)$ :

$$
\begin{gathered}
\frac{2 c \rho R^{8}}{7} \frac{d a}{d t}-\frac{7 c \rho R^{5}}{20} \frac{d b}{d t}=-\frac{3 \lambda R^{6}}{5} a, \\
-\frac{7 c \rho R^{5}}{20} \frac{d a}{d t}+\frac{c \rho R^{2}}{2} \frac{d b}{d t}=0,
\end{gathered}
$$

решение которой с начальными условиями (9) имеет следующий вид:

$$
a(t)=a_{0} \exp (-k t), b(t)=0,7 a_{0} R^{3} \exp (-k t)-0,7 a_{0} R^{3}+b_{0},
$$

где $k=280 \lambda /\left(19 c \rho R^{2}\right)$.

Для примера были проведены расчеты при следующих значениях параметров: $R=0,06 \mathrm{м}$;

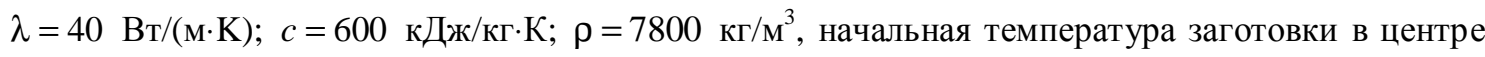
сечения $-T_{1}=1100{ }^{\circ} \mathrm{C}$, на поверхности $-T_{2}=500{ }^{\circ} \mathrm{C}$. Приведенные на рис. 1 . графики изменения температуры в центре сечения и на поверхности иллюстрируют выравнивание температуры заготовки по сечению.

Алгоритм решения термомеханической задачи. Рассмотрим теперь случай, когда параллельно с тепловым процессом, описываемым задачей (6), цилиндрическая заготовка подвергается деформированию. Будем считать материал заготовки идеально пластическим, причем его свойства зависят от температуры:

$$
\tau_{s}=\tau_{0}+\mu \theta
$$

Цилиндр подвергается радиальному обжатию, описываемому граничным условием:

$$
f_{r}=f_{*} .
$$

Здесь $\tau_{0}$ и $\mu$ - параметры материала, $f_{r}-$ радиальная компонента поверхностного напряжения в полярной системе координат, $f_{*}$ - заданное напряжение обжатия. 


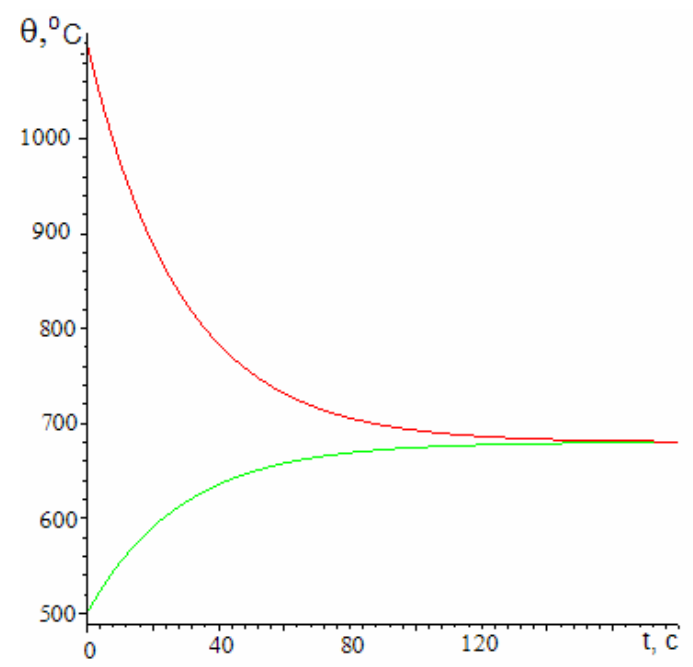

Р и с. 1. Зависимость температуры характерных точек заготовки от времени:

1 - температура центра; 2 - температура поверхности

Чтобы описать изменение температуры в заготовке в процессе деформирования, необходимо рассмотреть связную термомеханическую задачу. Функционал вариационного принципа виртуальных скоростей [1-3], соответствующий деформационной части задачи, выглядит следующим образом:

$$
J=\int_{0}^{R}\left(\tau_{s} \mathrm{H}^{\prime}+\rho w_{r} v_{r}^{\prime}\right) r d r+\left.R f_{*} v_{r}^{\prime}\right|_{r=R},
$$

где $v_{r}$ - компонента вектора скорости, $w_{r}-$ компонента вектора ускорения, $\mathrm{H}$ - интенсивность скоростей деформации сдвига, $\mathrm{H}=\sqrt{2 e_{i j} e_{i j}}, \quad e_{i j}-$ компоненты девиатора скоростей деформации. Функционал (7) с учетом выделения тепла при пластической деформации примет вид

$$
J_{\theta}=\int_{0}^{R}\left(\frac{\lambda}{2}\left(\frac{\partial \theta^{\prime}}{\partial r}\right)^{2}+c \rho \circledast \theta^{\prime}-\tau_{s} \mathrm{H} \theta^{\prime}\right) r d r .
$$

Примем виртуальное поле температуры в виде (8), а виртуальное поле скоростей - в виде:

$$
v_{\varphi}^{\prime}=0, v_{r}^{\prime}=u r
$$

где в каждый момент времени значение $u$ - варьируемый параметр. Таким образом, функционалы (15) и (16) выражаются через параметры $a, b, u$. Отметим, что при решении нестационарных задач деформирования безусловно надо учитывать формоизменение тела, например, используя лагранжевы координаты. Однако, учитывая малость деформаций, а также тестовый характер расчетов, мы в данной задаче этим пренебрегаем.

Дальнейшее решение производится аналогично рассмотренной ранее задаче. В необходимые условия экстремума функционалов (15) и (16) подставляются соответственно значения ускорения $w_{r}=\frac{d u}{d t} r$ и производной от температуры (10). В результате получается система трех дифференциальных уравнений относительно функций $a(t), b(t)$ и $u(t)$ :

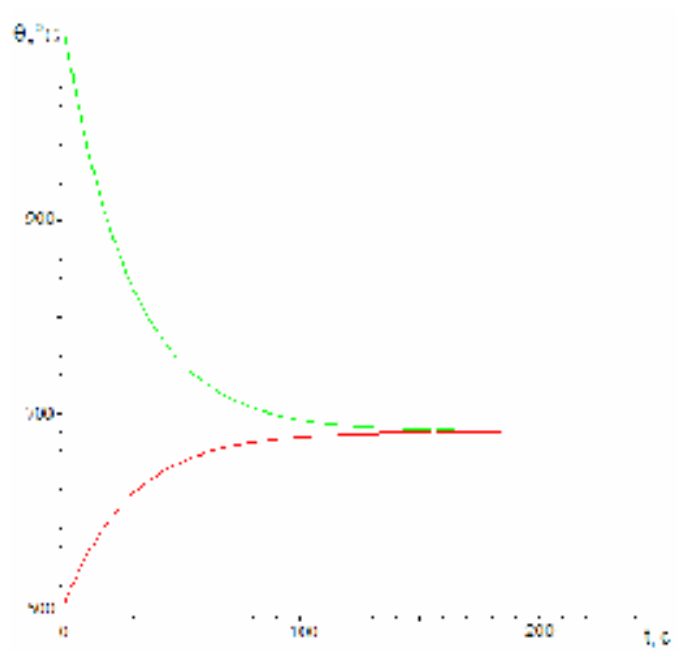

Р и с. 2. Изменение температуры для термомеханической задачи: 1 - температура центра; 2 - температура поверхности

$$
\begin{aligned}
\frac{d a}{d t} & =-\frac{2 a\left(19 \mu R^{2} u+420 \lambda\right)}{57 c \rho R^{2}}, \\
\frac{d b}{d t} & =-\frac{2\left(294 \lambda R a+19 \tau_{0} u+19 \mu b u\right)}{57 c \rho}, \\
\frac{d u}{d t} & =\frac{-7 \mu R^{2} a+10 \mu b+10 \tau_{0}-30 f_{*}}{10 \rho R^{2}} .
\end{aligned}
$$

Тестовые расчеты были проведены для образца с теми же параметрами и условиями, что и в примере из предыдущего пункта. Значения механических параметров были приняты следующими: $\tau_{0}=2 \cdot 10^{8}$ Па, $\mu=-1,7 \cdot 10^{5}, f_{*}=3,5 \cdot 10^{7}$ Па. Система уравнений (18) была решена численно. На рис. 2 показаны графики изменения температуры для точки поверхности и центра сечения, лежащей на оси цилиндра. Они не сильно отличаются

от графиков на рис. 1, что соответствует виду уравнений (18) и может быть объяснено малостью деформаций. 


\section{БИБЛИОГРАФИЧЕСКИЙ СПИСОК}

1. Колмогоров В. Л. Метод расчета НДС в общей краевой задаче развитого течения // Вестник ПГТУ. Механика, 1995. - № 2. - C. 87-98.

2. .Kolmogorov V. L, Fedotov V. P., Spevak L. F. A mathematical model for the formation and development of defects in metals // Studies in Applied Mechanics. №45. Advanced Methods in Materials Processing Defects. Elsevier, 1997. Pp. 51-60.

3. Kolmogorov V. L., Spevak L. F., Gorshkov A. V. A method for calculating the stress-strain state in the general boundary value problem of metal forming - part 2 // Int. J. of Solids and Structures. 1999. №36. Pp. 1263-1275.

4. Федотов В. П., Спевак Л. Ф. Решение динамических задач пластичности основанным на вариационной постановке методом разделения переменных // Математическое моделирование, 2000. — Т. 12, №7. — С. 36-40.

Работа выполнена при поддержке РФФИ, проект № 04-01-00274.

Поступила 26.12.2005 2.

УДК 539.376

Я. М. Клебанов, И. Е. Адеянов, Е. И. Ладягина

\section{ЧИСЛЕННЫЙ АНАЛИЗ ПОЛЗУЧЕСТИ КОНСТРУКЦИЙ ПРИ СЛОЖНОМ НАГРУЖЕНИИ}

Рассматривается процедура численного решения задач ползучести конструкций при сложном нагружении. Определяющие уравнения принимаются в виде, учитывающем начальную анизотропию реологических свойств и комбинированное деформационное упрочнение материала. Пошаговая прочедура интегрирования уравнений краевой задачи опирается на модифицированный метод Эйлера. Она встраивается как пользовательская в СAE ANSYS. Приводятся примеры расчётов двух конструктивных элементов. Анализируются закономерности ползучести конструкиий при сложном нагружении.

Введение. В последние пятнадцать лет происходит интенсивное развитие численных методов анализа напряжённо-деформированного состояния твёрдых тел в условиях ползучести. Накопленный в этой области опыт свидетельствует о том, что решение задач ползучести связано с большими трудностями, чем анализ упругопластического поведения, поскольку определяющие уравнения ползучести являются обычно более сложными. В особенности это относится к условиям непропорционального изменения внешних нагрузок, когда процесс деформирования существенно зависит от истории нагружения. Последнее значительно усложняет вид определяющих уравнений, применяемых для описания возникающих здесь эффектов. За последнее десятилетие такие зависимости рассматривались в работах [1-7].

Большинство существующих профессиональных коммерческих программных пакетов анализа напряжённо-деформированного состояния твёрдых тел позволяет пользователю встраивать в них новые определяющие уравнения. Поэтому весьма важной представляется возможность эффективной реализации разрабатываемой модели материала в составе таких пакетов альтернативой является создание новой программы, что связано с большими финансовыми и временными затратами.

Единственным широко используемым методом решения краевых задач деформирования является метод конечного элемента (МКЭ) - он применяется в большинстве профессиональных программных средств. Приложение МКЭ к задачам ползучести осуществляется путём пошагового интегрирования уравнений краевой задачи. Оно затруднено высокой нелинейностью и “жёсткостью” получаемых зависимостей [8]. Стабильность и точность МКЭ в этом случае существенно зависит от выбора временного шага и метода интегрирования.

Метод, альтернативный шаговым процедурам, был предложен в работах [9-11]. Решение ищется путём построения итераций сразу на всём заданном временном интервале. Приращение искомых величин: напряжений, деформаций и др. представляется в виде сумм произведений функций времени и координат, последовательно уточняемых на каждой из итераций в процессе решения линеаризированных краевых задач. Полученные оценки показывают, что в случае ползучести при сложном нагружении этот подход уступает по эффективности существующим шаговым процедурам. 